\title{
Pediatric SCIWORA-Type Injuries Revisited: What Should be the Most Discerning Definition of SCIWORA at the Current Days?
}

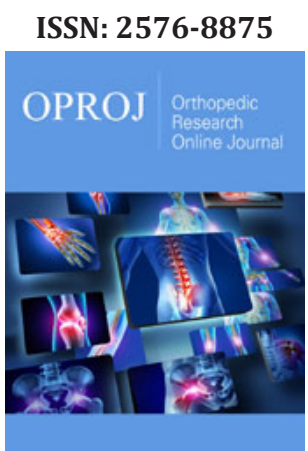

*Corresponding author: Elisa LópezDolado, Rehabilitation Department and Laboratory of Interfaces for Neural Repair (LINER), Hospital Nacional de Parapléjicos, Toledo, Spain

Submission: 梅 September 14, 2021 Published: 监September 21, 2021

Volume 8 - Issue 5

How to cite this article: Raquel Madroñero-Mariscal, Elisa LópezDolado*. Pediatric SCIWORA-Type Injuries Revisited: What Should be the Most Discerning Definition of SCIWORA at the Current Days?. Ortho Res Online J. 8(5). OPROJ. 000697. 2021.

DOI: 10.31031/OPROJ.2021.08.000697

Copyright@: Elisa López-Dolado, This article is distributed under the terms of the Creative Commons Attribution 4.0 International License, which permits unrestricted use and redistribution provided that the original author and source are credited.

\section{Raquel Madroñero-Mariscal ${ }^{1}$ and Elisa López-Dolado ${ }^{2,3 *}$}

${ }^{1}$ Department of Physiatrist \& Rehabilitation, Fundación del Lesionado Medular \& Hospital Universitario Infanta Leonor, Spain

${ }^{2}$ Clínical Chief, Department of Physiatrist \& Rehabilitation and Laboratory of Interfaces for Neural Repair (LINER), Hospital Nacional de Parapléjicos, Spain

${ }^{3}$ Associate Professor, Department of Medicine and Medical Specialities, School of Medicine, Universidad de Alcalá (UAH) Spain

\begin{abstract}
The traditional concept of "SCIWORA" is nowadays being reassessed due to the accumulation of clinical knowledge and the improvement in diagnostic accuracy to study this entity. In the current era of magnetic resonance imaging as the gold standard in spinal pathology, particularly the diffusion tensor imaging scanning, the present article aims to respond the challenge of critically reviewing and redefining this entity as accurately as possible. Another important fact will be to discuss the best treatment option for these patients, because while conventional approaches agreed with a conservative management, some modern streams of thought defend an early surgical procedure to avoid a worst prognosis in selected cases.
\end{abstract}

Keywords: SCIWORA; Paediatric spinal cord trauma; Children; Magnetic resonance imaging; Diffusion tensor imaging; Spinal cord concussion; Spinal cord infarctions

Abbreviations: ALL: Anterior Longitudinal Ligament; CNS: Central Nervous System; CT: Computed Tomography; DHb: Deoxygenated Hemoglogin; DTI: Diffusion Tensor Imaging; MRI: Magnetic Resonance Imaging; MHb: Methemoglobin; PLL: Posterior Longitudinal Ligament; PTS: Post-Traumatic Syringomyelia; SCI: Spinal Cord Injury; SCInf: Spinal Cord Infarctions

\section{Introduction}

Since the 1980s Pang, Wilberger and Pollack described a rare type of spinal cord injury (SCI) in children in which no evidence of spinal cord injury was found [1,2], the accumulation of clinical knowledge and the improvement in diagnostic accuracy have allowed the biomechanics, pathophysiology, and treatment of this entity to be reassessed. Over the three decades since its description, the power of the spinal pathology diagnosis has exponentially increased due to the widespread use of Magnetic Resonance Imaging (MRI). Thanks to MRI, it is not only possible to determine much more accurately the extent and severity of spinal cord damage, but also the presence of extramedullary lesions "blind" to plain X-ray or even tomography scans, so the proper definition of the SCIWORA has been challenged and thus its existence has been called into question.

With that in mind, in the era of MRI as the gold standard in spinal pathology, the present article aims to respond the challenge of critically reviewing and redefining this entity as accurately as possible.

\section{A historical perspective}

By the time Pan and co-workers coined the term SCIWORA, several other authors had described the distinctive profile of the pediatric spinal trauma from that of adults and the major anatomical and biomechanical differences in the pediatric spine that underlie it. Since paediatric spinal trauma is fortunately rare [3-5], it is very commendable that in the late 1960s and 1970s several authors collected and published sufficiently large patient series to be able to elucidate the types of traumatic SCI, being SCIWORA just one of them, the injury mechanisms underlying, the management and the final outcome. Among them, it is worth mentioning David C Burke's [6,7] works, who published in 1971 and 1974 respectively two paediatric 
spinal trauma series -children under 13 years of age- characterized by the contrast between the absence or paucity of vertebral involvement signs and the severity of the spinal cord damage. It was thus postulated that the elastic, cartilaginous spine of a young child may be grossly distorted by flexion/rotation/distraction forces but does not disrupt through its inherent elasticity. The spinal cord, however, is unable to withstand such stretching forces, and a longitudinal spinal damage may result. The case series published by Hachen [8] in 1977, Andrews [9] in 1979 and Anderson [10] in 1980 support and confirmed the abovementioned data and raised other relevant and surprisingly current questions such as the most appropriate therapeutic approach at the time of acute SCI or the functional prognosis in the chronic phase depending on the SCI type, level and severity.

Two decades after that, Ghatan \& Ellenbogen, in a thorough review of the epidemiology, mechanisms of injury, developmental radiographic anatomy, and neuropathology of inflicted pediatric spine and SCI, reflect on the huge impact on children growing up with SCI, their families, the health care providers, and the society in general [11]. But it was Pang himself who, in 2004, reviewed his own case series over two decades since he defined SCIWORA, including the information provided by MRI both to define spinal cord damage and to elucidate soft tissue damage to confirm or exclude vertebral instability. In that work, Pang proposed the three clinical features that are still today associated with this type of injury: 1) occult instability and 2) delayed and/or 3) recurrent SCI, being the last two a consequence of the first one [12].

At the dawn of the $21^{\text {st }}$ century, the term SCIWORA had caught on [13], although the idea remains that the higher the resolution of imaging for spinal damage, especially MRI, the lower the number of true cases of SCIWORA [14]. Furthermore, the less spinal cord damage the initial MRI shows, the less severe the clinical manifestations of SCI and the better the prognosis [15]. But on top of all the above, probably the greatest value of the work of Pang et al. has been to help to raise the suspicion and therefore to make the diagnosis of these rare but potentially fatal type of injuries.

\section{Current diagnosis and prognosis of SCIWORA-type injuries}

Sports, motor vehicle collisions, falls, assaults and being beaten, are traumatic injury mechanisms [16], that should make physicians think of a SCIWORA injurie cause of paediatric SCI, whenever a child shows signs of muscle weakness, sensory loss, bowel and/or bladder sphincter control loss, or reflexes loss.

An initial radiograph and Computed Tomography (CT) scan are initially recommended to be done [12,16-18]. However, those imaging tests will not find any pathological changes in these patients. For this reason, it should be practiced an MRI, in order to exclude other anatomical disruptions that affect the osteomuscular system that can damage the biomechanical and anatomical structure of the different stabilization capsules, ligaments and bone regions of the spine, which would explain the cause of those symptoms.
If we consider our current definition of SCIWORA as the paediatric SCI which appears as a consequence of a traumatic injury, showing clinical signs and symptoms of spinal cord damage without abnormalities showed at the available imaging tests, we can guess that MRI, during the past decades, has become the gold standard to exclude other traumatic leading causes of SCI, different from SCIWORA. Therefore, MRI has not only become the acutest tool to achieve the final diagnosis of SCIWORA, but also to stand the treatment management and prognosis of each type of injury, according to its findings or even "no findings" showed [12,17]. In this way, MRI has been able to demonstrate the visualization of the damage of the soft tissues that explains the biomechanical and anatomical disruption of the spine. Moreover, MRI development has not only provided with a before' and an after' in the way of diagnosing this entity, but also has reached a way to make a better prognosis.

Because of its implication on the management and outcome, and because of the strong correlation of its traumatic injury cause, those damages are detailed below:

Extraneural abnormalities: the rupture of the Anterior Longitudinal Ligament (ALL), that can cause a retrolisthesis of the upper vertebral segment, is associated with extension injuries; the rupture of the Posterior Longitudinal Ligament (PLL), is associated with hyperflexion injuries; tectorial membrane hemorrhage, is associated with child abuse; and hemorrhages and edema in the interspinous and interlaminar ligaments are associated with distraction and flexion injuries. According to our current SCIWORA definition, those findings would exclude SCIWORA, as they would anatomically explain the reason of neurological symptoms of the SCI. However, there is also a new insight that physicians should keep in mind in order to stablish the correct diagnosis of SCIWORA, because, thought MRI can be able to detect the previous injuries within the first 24 hours of the lesion, it may be possible too that those changes may go unnoticed at that time, which make physicians to consider the necessity of repeating the MRI during the following days [17]. This occurs because the most common MRI which are available at hospitals detect fascial, muscular and fat planes with high signal, which can mask the finding of small sources of methemoglobin (MHb). The use of fat-suppression sequences which reduces the high signal of fat but not blood on short-TR (T1weighted) images, can help to highlight the soft tissue hemorrhages in order to correctly discard the diagnosis of SCIWORA [12].

Neural abnormalities: the intramedullary damage due to a traumatic mechanism causing signs and symptoms of SCI, can also anatomically explain the damaging process of this type of injury, thought a biomechanical impairment will not be the leading cause of its malfunction, but a direct intraneural damage will do it. In those cases, as anatomical structural damage can be observed, the diagnosis of SCIWORA will be excluded. However, the authors of the present work have considered that those changes must be addressed in this work because of its playing roll in reaching the correct management and stablishing a prognosis of these patients. 
In this way, the initial MRI can detect the deoxygenated hemoglogin (DHb) up to 8 days after injury. Beyond 3 days after injury, short-TR MRI can detect intracellular MHb because it shows an hyperintense image, and extracellular MHb because it shows an hyperintense image on both short and long-TR images. Finally, extracellular MHb will be transformed in hemosiderin deposits for the rest of the patient's life, which could be shown as hypointense images on both short and long-TR images as well [12]. Besides, the development of the Diffusion Tensor Imaging (DTI) studies has brought the opportunity to identify the lesions of motor and sensory tracts, cephalad and caudal to the lesion, which has demonstrated some good correlation with the ISNCSCI total motor score and light touch sensory score. Another interesting contribution of this type of imaging study is that it provides a substantial information about the grade of injury of the neural tissue above and below the level of the lesion, which is difficult to guess just with the clinical examination [19]. Depending on the initial MRI findings, the prognosis will be different. When a complete disruption of the spinal cord, or a major hemorrhage (more than $50 \%$ of the cross-sectional area of the cord on axial MRI) is affected, a worst prognosis due to severe neural deficits should be expected. Meanwhile, when minor cord hemorrhage (less than $50 \%$ of the cross-sectional area of the cord on axial MRI) is initially observed, a better prognosis including partial recovery can be expected. If the initial MRI shows only edema, it seems reasonable to assume good outcomes. Finally, patients who only show alteration in electrophysiological proofs or clinically symptoms, but no alterations are found in MRI, are expected to develop the better prognosis with full recovery $[12,17]$.

\section{Controversies and future perspectives}

Even if the definition of SCIWORA may be somewhat different from one study to another, there are currently no discrepancies between authors regarding the differential nature of the paediatric spinal trauma with respect to the adult one. Nor is there any doubt that MRI is the gold standard imaging test for its diagnosis, because it is able to provide essential information to define the type of injury and determine its extent and prognosis. Still, until recently, there was even consensus on the initial therapeutic approach, but not anymore, because as we shall explain below, conventional conservative management has recently been called into question.

There is no consensus about the benefit of using corticoids at the acute phase of the injury. While some authors recommend using intravenous steroid therapy even before MRI scan can be practiced $[12,17]$, there are others that support a lack of evidence in their use [16], principally because of the risk of hypoperfusion of the spinal cord due to its use, which could be the cause of secondary injuries in spinal cord trauma [17]. Traditionally, most of the authors agreed with a conservative approach for the treatment of this entity, by practicing the immobilization with orthoses of the involved segment for 3 months and avoiding every sports' activity for 6 months [17]. However, nowadays, there are some other authors like MartínezPérez [20], Fehlings [21] and Qi [22], who call into question this assumption, sustaining that an early surgical management can avoid a worst prognosis. Moreover, Qi et al. [22] explain that a prompt surgical treatment (within the first 3-7 days after injury) can achieve significant and quicker improvements measured by the ASIA scale in both short-term and long-term follow-up effects. As the current studies which explore this area tend to dispose of a poor sample size, it seems reasonable to wait for much larger sample sizes works to be able to standardize the best procedure for the initial management of the treatment of SCIWORA, especially when the mildest findings such as edema or even no alterations are found at MRI, which could represent the more controversial cases to undergo surgery at the early beginning of the lesion. Figure 1 summarizes the diagnosis and therapeutic approach algorithm for SCIWORA-type injuries.

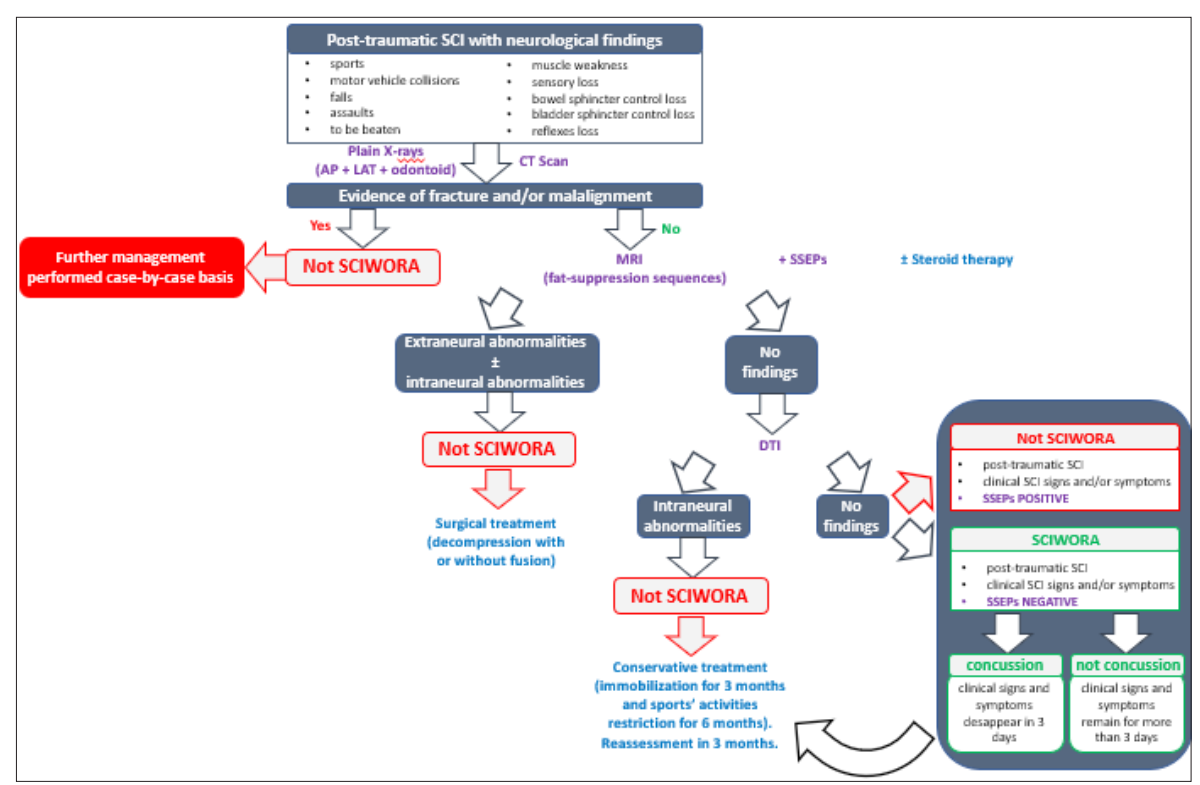

Figure 1: Algorithm of diagnosis and treatment of SCIWORA (modified from Konovalov et al. [16]). SCI: spinal cord injury; MRI: magnetic resonance imaging; SSEPs: somatosensory evoked potentials; DTI: diffusion tensor imaging. 
Another important point of controversy is whether or not to include in the SCIWORA series those patients with mild, subtle or even evanescent neurological symptoms and/or those with no obvious damage on initial MRI. In all these cases, full recovery is to be expected. Some authors establish the differences between what they consider "real SCIWORA" and a spinal cord "concussion" by presuming that clinical signs and symptoms of SCI concussion disappear within 3 days, while they remain for more than 3 days in "real SCIWORA" [22]. Nevertheless, for us, the concept of "SCIWORA" will not be determined by the clinical aspect, but by the imaging instrumental test detection's capacity, therefore, taking for granted that spinal concussion should be considered a SCIWORAtype injury. This is not a trivial aspect, as differences have even been described in relation to the medical speciality of the physician making the patient classification at the time of the acute SCI [23]. The prevalence of SCIWORA has been reported in a wide range between $4 \%$ and $67 \%[12,14,17]$ of pediatric spinal trauma cases, a large hairpin that could be at least partially explained by variability in the population studied, but more likely owing to the great disparities in the SCIWORA definition [14]. It is our understanding, as it was for Pang and co-workers [12], that it is of great importance taking these subtle cases into account and carefully follow their evolution up, if only for two reasons: first, the potential impact on somatic growth and thus the development of subsequent orthopaedic deformities. The vast majority of SCIWORA cases affect patients under 6 years of age and are located in the cervical spine [12-15,17], i.e. patients in the process of growth and development. Since the negative impact of Central Nervous System (CNS) injuries on somatic growth in children is well established [24], even mild neurological sequelae suffered at a young age will have repercussions on orthopaedic development in the medium to long term [25], especially progressive neuromuscular scoliosis [26]. Second, the possibility of the development of intraspinal tissue damage resulting from the development of gliosis and/or syringomyelia [27] leading to unexpected and unexplained long-term neurological impairment, even in the case of only transient or mild initial neurological signs and symptoms have been addressed both among pediatric [28,29] and adult population [30]. Syringomyelia is recognized as the main factor of neurological deterioration following SCI, since MRI has improved the diagnosis, but the correlation between the severity of SCI and the incidence of Post-Traumatic Syringomyelia (PTS) or the time interval to its onset is controversial [30]. Among paediatric population, the presence of syringomyelia is rarely reported, but progressive scoliosis, as it is the case of the neuromuscular scoliosis secondary to pediatric SCI [26], stands out as a possible isolated manifestation [31]. So, it seems reasonable also to follow the evolution of those patients with mild or evanescent SCIWORA-type lesions until we have ascertained that there are no repercussions, i.e. scoliosis or PTS, attributable to the initial SCI.

It also remained controversial whether or not spinal cord infarctions (SCInf) triggered by a slight trauma or an effort should be considered SCIWORA-type cases or not. With respect to the spinal damage mechanism, both entities show different degrees of spinal cord ischaemia, but while in SCIWORA-type lesions it is secondary to spinal cord distraction [12], in SCInf it is often due to vasospasm or embolic event of the anterior spinal artery [32,33]. As for the clinical presentation, SCInf is characterized by the sudden onset of pain and progressive neurological deterioration over the first 24 hours [33], while deferred neurological symptoms in SCIWORA, usually paralysis or paresis, appears with a latency of between 30 minutes and several days, preceded at the time of acute SCI by weakness or Lhermitte's sign [12]. The triggering trauma is usually of higher energy in SCIWORA than in SCInf, but the severity of the resulting SCI and the MRI findings can be similar and have been related to the biomechanical immaturity of the pediatric spine [34], so perhaps it may be the same pathogenic phenomenon but to a different degree.

\section{Conclusion}

In the MRI era, it makes sense that SCIWORA-type lesions might be restricted to the cases of SCI without neuroradiologic abnormalities [23]. Nowadays there is consensus on the distinctive profile of the paediatric spinal trauma from that of adults and the major anatomical and biomechanical differences in the paediatric spine that underlie it, as much as on the great value of more classical approaches like that of Pang and co-workers in helping to raise the suspicion and therefore facilitate the diagnosis of these rare but potentially fatal type of injuries. MRI is the gold standard diagnostic tool, particularly the DTI scanning, capable of identifying microstructural changes and tract damage. Most of the authors agreed with a conservative approach for its treatment, but some others propose an early surgical management to avoid a worst prognosis in selected cases.

\section{Acknowledgement}

Author's role: All authors had significant and substantial roles in the conception, analysis, interpretation, and writing of this report.

\section{Conflict of Interest}

No funding was secured for this work. All authors declare that they have nonfinancial interests that might be relevant to the submitted work.

\section{References}

1. Pang D, Wilberger JE (1982) Spinal cord injury without radiographic abnormalities in children. J Neurosurg 57:114-129.

2. Pang D, Pollack IF (1989) Spinal cord injury without radiographic abnormality in children: The SCIWORA syndrome. J Trauma 29: 654664.

3. Kim C, Vassilyadi M, Forbes JK, Moroz NWP, Camacho A, et al. (2016) Traumatic spinal injuries in children at a single level 1 pediatric trauma centre: report of a 23-year experience. J Can J Surg, 59(3): 205-212.

4. Meinig H (2020) Diagnostics and treatment of cervical spine trauma in pediatric patients: Recommendations from the Pediatric Spinal Trauma Group. Der Unfallchirurg 123(4): 252-268. 
5. Beckmann NM, Chinapuvvula NR, Zhang X, Clark WO (2020) Epidemiology and imaging classification of pediatric cervical spine injuries: 12-year experience at a level 1 trauma center. AJR 214: 13591368.

6. David C Burke (1971) Spinal cord trauma in children. Spinal Cord 9:1-14

7. David C Burke (1974) Traumatic spinal paralysis in children. Spinal Cord 11: $268-276$.

8. Hachen HJ (1977) Spinal cord injury in children and adolescents: diagnostic pitfalls and therapeutic considerations in the acute stage. Spinal Cord 15:55-64.

9. Andrews LG, Jung SK (1979) Spinal cord injuries in children in British Columbia. Spinal Cord (17): 442-451.

10. Anderson JM, Schutt AH (1980) Spinal injury in children: a review of 156 cases seen from 1950 through 1978. Mayo Clinic Proceedings 55(8): 499-504.

11. Ghatan S, Ellenbogen RG (2002) Pediatric spine and spinal cord injury after inflicted trauma. J Neurosurgery 13(2): 227-233.

12. Pang D (2004) Spinal cord injury without radiographic abnormality in children, 2 decades later. Neurosurgery 55(6): 1325-1343.

13. Rolfe K, Beck A, Kovach T, Mayeda B, Liu C (2019) Non-locality and the misdiagnosis of spinal cord injury without radiographic abnormality: proof of concept. Spinal Cord Series and Cases 5: 11-21.

14. Mahajan P, Jaffe DM, Olsen CS, Leonard JR, Nigrovic LE, et al. (2013) Spinal cord injury without radiologic abnormality in children imaged with magnetic resonance imaging. J Trauma Acute Care Surg 75(5): 843847.

15. Martínez-Pérez R, Joswig H, Rayo N, Bertazzo-Silveira G (2020) Spinal cord injury without radiological abnormalities and the importance of magnetic resonance: not only diagnostic, but also predictive! Acta Biomed 91(3): e2020084.

16. Atesok K (2018) Posttraumatic spinal cord injury without radiographic abnormality. Advances in Orthopedics 2018: 7060654.

17. Konovalov N (2020) Pediatric cervical spine injuries and SCIWORA: WFNS spine committee recommendations. Neurospine 17(4): 797-808.

18. Copley PC, Tilliridou V, Kirby A, Jones J, Kandasamy J (2019) Management of cervical spine trauma in children. European Journal of Trauma and Emergency Surgery: Official Publication of the European Trauma Society 45(5): 777-789.

19. Saksena S (2019) Diffusion tensor imaging assessment of regional white matter changes in the cervical and thoracic spinal cord in pediatric subjects. Journal of Neurotrauma 36(6): 853-861.

20. Martinez-Perez R, Munarriz PM, Paredes I, Cotrina J, Lagares A (2017) Cervical spinal cord injury without computed tomography evidence of trauma in adults: Magnetic resonance imaging prognostic factors. World neurosurgery 99: 192-199.

21. Fehlings MG (2012) Early versus delayed decompression for traumatic cervical spinal cord injury: results of the Surgical Timing in Acute Spinal Cord Injury Study (STASCIS). PloS one 7(2): e32037.

22. Qi C, Xia H, Miao D, Wang X, Li Z (2020) The influence of timing of surgery in the outcome of Spinal Cord Injury Without Radiographic Abnormality (SCIWORA). Journal of Orthopaedic Surgery and Research 15(1): 223.

23. Yucesoy K, Yuksel KZ (2008) SCIWORA in MRI era. Clin Neurol Neurosurg 110(5): 429-433.

24. Deng L, Li G, Rao B, Li H (2015) Central nervous system-specific knockout of Brg1 causes growth retardation and neuronal degeneration. Brain Res 1622: 186-195.

25. Parent S, Dimar J, Dekutoski M, Roy-Beaudry M (2010) Unique features of pediatric spinal cord injury. Spine 35(21S): S202-S208.

26. Cuddihy LA, Antonacci MD, Hussain AK, Vig KS, Mulcahey MJ, et al. (2019) Progressive neuromuscular scoliosis secondary to spinal cord injury in a young patient treated with nonfusion anterior scoliosis correction. Top Spinal Cord Inj Rehabil 25(2): 150-156.

27. Kleindienst A, Engelhorn T, Roeckelein V, Buchfelder M (2020) Development of pre-syrinx state and syringomyelia following a minor injury: a case report. J Med Case Reports 14: 223.

28. Nagasawa H, Ishikawa K, Takahashi R, Takeuchi I, Jitsuiki K, et al. (2017) A case of real spinal cord injury without radiologic abnormality in a pediatric patient with spinal cord concussion. Spinal Cord Series and Cases 3: 17051.

29. Sakti YM, Saputra MA, Rukmoyo T, Magetsari R (2018) Spinal Cord Injury Without Radiological Abnormality (SCIWORA) manifested as selflimited brown-SEQUARD syndrome. Trauma Case Reports 18: 28-33.

30. Ullah S, Qureshi AZ, Tantawy SS, AlJaizani YA (2020) An unusual mechanism of spinal cord injury due to active neck stretching and its functional implications. Clin Case Rep 8: 1090-1093.

31. Karam Y, Hitchon PW, Mhanna NE, He W, Noeller J (2014) Post-traumatic syringomyelia: outcome predictors. Clin Neurol Neurosurg 124: 44-50.

32. Martínez-Albaladejo I, Procházková M, Pérez-Sebastián I, Cuesta B, Martínez-Ferrández C, et al. (2016) Syringomyelias in paediatrics: a retrospective study of 25 cases. Rev Neurol 63: 193-200.

33. Bar C, Cheuret E, Bessou P, Pedespan JM (2017) Childhood idiopathic spinal cord infarction: Description of 7 cases and review of the literature. Brain and Development 39(10): 818-827.

34. Sheikh A, Warren D, Childs AM, Russell J, Liddington M, et al. (2017) Paediatric spinal cord infarction-a review of the literature and two case reports. Childs Nerv Syst 33: 671-676. 